\title{
Simulation of sigmoid structure and filament eruption of AR11283 using a three-dimensional data-driven magnetohydrodynamic model
}

\author{
S. T. Wu ${ }^{1}$, Chaowei Jiang ${ }^{2}$, Xueshang Feng ${ }^{2}$, Qiang $\mathbf{H u}^{1}$, and \\ Yang $\mathrm{Liu}^{3}$ \\ ${ }^{1}$ Center for Space Plasma and Aeronomic Research, University of Alabama in Huntsville, \\ Huntsville, AL 35805, USA \\ email: wus@uah.edu \\ ${ }^{2}$ SIGMA Weather Group, State Key Laboratory for Space Weather, Center for Space Science \\ and Applied Research, Chinese Academy of Sciences, \\ Beijing, 100190, China \\ ${ }^{3}$ W.W. Hansen Experimental Physics Laboratory, Stanford University, \\ Stanford, CA 99305, USA
}

\begin{abstract}
This paper describes an MHD simulation of an observed Sigmoid in AR 11283 from its formation to eruption. The Non-linear Force Free MHD model (Jiang and Feng, 2012) and the data-driven active region evolution model (Wu et al., 2006; Jiang et al. 2013) together with the SDO/HMI magnetograms are used. We show the successful simulation results of the eruption of a flux-rope structure.
\end{abstract}

Keywords. Sigmoid, MHD Simulation, Active Region, Magnetogram

Sigmoids are generally observed in the solar corona in soft X-ray. It has been shown observationally (Canfield et al. 1999, 2007) that active regions with sigmoidal morphology are more likely to produce eruptive events. In this scenario, we will analyze a recent observed active region (AR11283) using a three-dimensional data-driven magnetohydrodynamic (MHD) active region evolution model (Wu et al. 2006; Jiang et al. 2012) together with a nonlinear force-free model (Jiang \& Feng, 2012) for the construction of the initial state with the presence of a sigmoidal feature. It has been recognized that the AR11283 is a very productive source region, producing a number of flares and CMEs when it was located near disk center. In the present study, we will focus on the initiation process of a CME on 6 September 2011 accompanied by a sigmoid eruption around 22:20UT. To carry out this simulation, we first have input the measured vector magnetic field on the photosphere
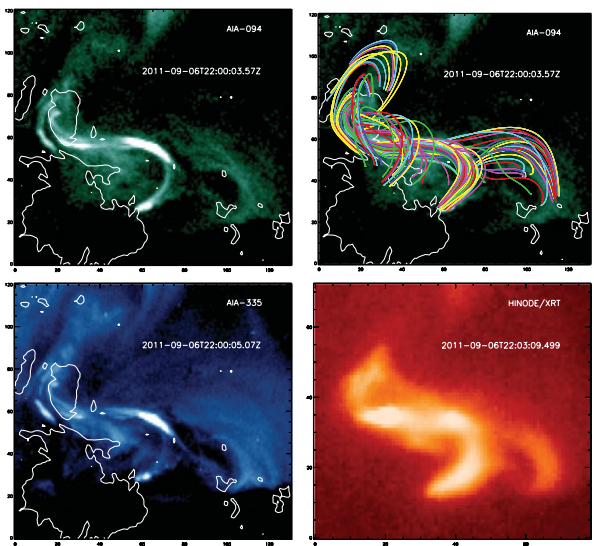

Figure 1. Pre-eruption MHD equilibrium state in comparison with observations for AR11283. Counterclock-wise from upper left panel: AIA-094, AIA-335, and Hinode/XRT images, and the simulated magnetic field lines with the AIA-094 image as the background. 

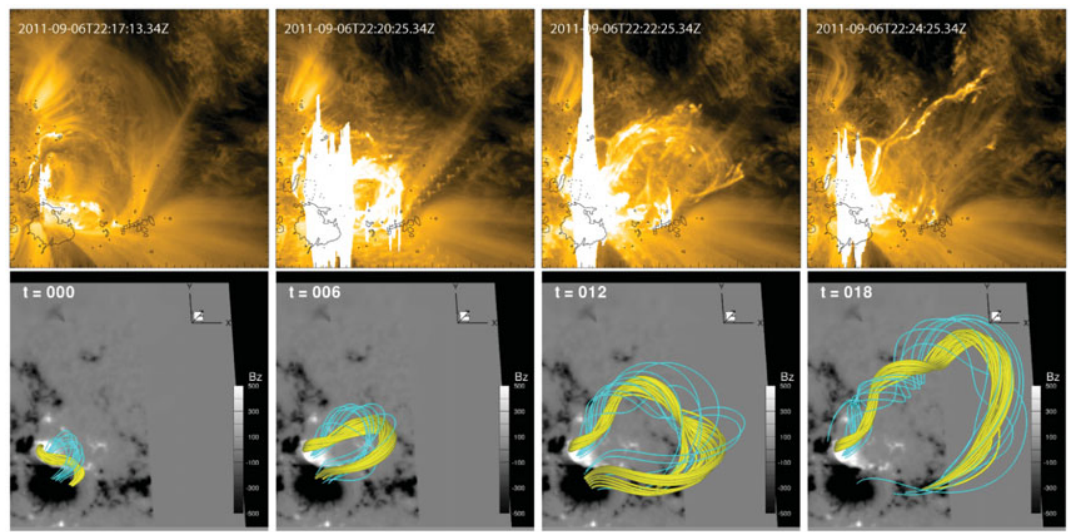

Figure 2. The MHD simulation result of the erupting flux-rope (twisted magnetic field-line) structure (bottom row) and the corresponding AIA-171 images at four different times.

into a nonlinear force-free model (Jiang \& Feng, 2012) to obtain the sigmoid structure before the eruption. Fig. 1 shows the simulated sigmoid at 2011-09-06 22:00UT immediately before the eruption. It is obvious that the simulated sigmoid matches the observations well. Then we embed this solution into our data-driven CESE-MHD model (Jiang et al. 2012), which results in an eruption as shown sequentially in Fig. 2. There the simulated eruption of the sigmoid compares remarkably well with the corresponding AIA imagest. The cause of the eruption is due to twist instability (Kliem \& Török, 2006; Török \& Kliem, 2007). We have ruled out the kink instability because we found that immediately above the sigmoid the magnetic field structure corresponding to part of a flux rope is strongly sheared and slightly twisted. Detailed discussion was given by Jiang et al. (2013a,b).

In summary, this is the first time an observed sigmoid from its birth to eruption has been successfully simulated based on vector magnetograms from SDO/HMI. A detailed description of the simulation results can be found in Jiang et al. 2013a,b.

\section{Acknowledgements}

This work is jointly supported by NSF (AGS-1153323; AGS-1062050) and NNSFC (41204126, 41231068, 41274192, 41031066, and 41074122). Data from observations are courtesy of NASA SDO/AIA and HMI science teams.

\section{References}

Canfield, R. C., Hudson, H. S., \& McKenzie, D. E. 1999, GRL, 26, 627

Canfield, R. C., Kazachenko, M. D., Acton, L. W., et al. 2007, ApJL, 671, L81

Jiang, C., Feng, X., Wu, S. T., \& Hu, Q. 2013a, ApJL, 771, L30

Jiang, C., Wu, S. T., Feng, X., \& Hu, Q. 2013b, ApJ, in press

Jiang, C., Feng, X., Wu, S. T., \& Hu, Q. 2012, ApJ, 759, 85

Jiang, C. \& Feng, X. 2012, ApJ, 749, 135

Kliem, B. \& Török, T. 2006, Physical Review Letters, 96, 255002

Török, T. \& Kliem, B. 2007, Astronomische Nachrichten, 328, 743

Wu, S. T., Wang, A. H., Liu, Y., \& Hoeksema, J. T. 2006, ApJ, 652, 800

$\dagger$ See https://dl.dropboxusercontent.com/u/96898685/Eruption_AR11283.gif for an animation. 